\title{
Safety and efficacy of an implantable device for management of gastroesophageal reflux in lung transplant recipients
}

\author{
Samantha E. Halpern ${ }^{1} \wedge$, Aryaman Gupta ${ }^{2,3}$, Oliver K. Jawitz ${ }^{2}$, Ashley Y. Choi ${ }^{1}$, Hai V. Salfity ${ }^{2}$, Jacob A. Klapper ${ }^{2}$, \\ Matthew G. Hartwig ${ }^{2}$ \\ ${ }^{1}$ School of Medicine, Duke University, Durham, NC, USA; ${ }^{2}$ Division of Cardiovascular and Thoracic Surgery, Department of Surgery, Duke \\ University Medical Center, Durham, NC, USA; ${ }^{3}$ School of Medicine, Johns Hopkins University, Baltimore, MD, USA \\ Contributions: (I) Conception and design: SE Halpern, A Gupta, HV Salfity, JA Klapper, MG Hartwig; (II) Administrative support: None; (III) Provision \\ of study materials or patients: HV Salfity, JA Klapper, MG Hartwig; (IV) Collection and assembly of data: SE Halpern, A Gupta, AY Choi; (V) Data \\ analysis and interpretation: SE Halpern, A Gupta, OK Jawitz, AY Choi; (VI) Manuscript writing: All authors; (VII) Final approval of manuscript: All \\ authors. \\ Correspondence to: Samantha E. Halpern, BA. Duke University Medical Center, Box 3863, Durham, North Carolina 27710, USA. \\ Email: samantha.halpern@duke.edu.
}

Background: Magnetic sphincter augmentation (MSA) is a promising minimally invasive surgical technique for management of gastroesophageal reflux disease (GERD); however, device implantation after transplantation has not been studied and may be concerning in these immunosuppressed patients. We explored the safety of the LINX Reflux Management System (MSA device) for management of GERD following lung transplantation (LTx).

Methods: Lung transplant recipients who underwent LINX implantation at our institution between 2017 and 2019 were followed prospectively in the Reflux Following Lung Transplantation and Associated Treatment Registry. Ambulatory $\mathrm{pH}$ testing and acid-suppressing medication use were compared before and after LINX implantation. One-year outcomes and change in pulmonary function were compared between matched LINX and fundoplication groups.

Results: Of 17 patients who underwent post-lung transplant LINX implantation, 8 (47.1\%) agreed to undergo post-LINX pH testing. Three/eight (37.5\%) patients achieved normal esophageal acid exposure time; $14(82.4 \%)$ remained on acid-suppressing medication at one-year under the direction of their transplant teams. One-year patient survival and change in pulmonary function were similar between groups. LINX patients experienced more early side effects.

Conclusions: Use of the LINX MSA device in a cohort of lung transplant recipients at our institution was associated with similar short-term safety compared to traditional fundoplication, however assessment of efficacy was limited. Further investigation is needed to characterize the long-term efficacy of LINX implantation after LTx.

Keywords: Gastroesophageal reflux disease (GERD); lung transplantation (LTx); LINX Reflux Management System

Submitted Nov 11, 2020. Accepted for publication Jan 29, 2021.

doi: $10.21037 /$ jtd-20-3276

View this article at: http://dx.doi.org/10.21037/jtd-20-3276

^ ORCID: 0000-0001-7316-1989. 


\section{Introduction}

Gastroesophageal reflux disease (GERD) is common after lung transplantation (LTx), affecting more than half of LTx recipients (1-3). In addition to typical lifestyle impacts of severe GERD, it may also predispose to micro-aspiration events that contribute to development of chronic lung allograft dysfunction (CLAD), a clinical manifestation of chronic rejection that accounts for significant morbidity and mortality after LTx (4). Pre-clinical studies support an association between GERD and CLAD, albeit not definitively, demonstrating increased inflammation and upregulation of pro-fibrotic mechanisms in lung allografts after aspiration events $(5,6)$. In the clinical setting, the presence of digestive components in bronchoalveolar lavage fluid (BALF) is also associated with heightened incidence and accelerated progression of CLAD $(7,8)$.

Laparoscopic anti-reflux surgery (LARS) has historically been the preferred treatment for post-LTx GERD (9). While anti-secretory medications such as proton pump inhibitors (PPI) raise gastric $\mathrm{pH}$, they do not reduce nonacid reflux events or digestive components in BALF $(9,10)$. Surgical GERD management provides a mechanical barrier to reflux and aspiration which may preserve pulmonary function, reduce CLAD severity (11-14), and improve symptom-related quality of life (15). While laparoscopic fundoplication is an effective treatment for post-LTx GERD, these procedures may be associated with side effects including chronic dysphagia, gas bloating, and inability to belch that may leave patients unsatisfied postoperatively $(16,17)$.

Magnetic sphincter augmentation (MSA) is an emerging minimally invasive surgical technique that relieves GERD symptoms, while avoiding potential side effects of traditional LARS procedures and shortening post-operative hospital stays (16). The LINX Reflux Management System is an MSA device composed of interlinked titanium beads with magnetic cores, placed laparoscopically around the gastroesophageal junction. The beads separate to accommodate a swallowed bolus and then reapproximate to augment the lower esophageal sphincter in the closed position (16). In the general population, the LINX device offers sustained decrease in mean acid exposure time, freedom from daily PPI use, and improved symptom-related quality of life (16,18-20). However, in immunosuppressed patients such as LTx recipients, device implantation is concerning due to risk of severe infection that may compromise long-term outcomes (21). We evaluated the safety and efficacy of the LINX Reflux Management System in this chronically immunosuppressed population. We present the following article in accordance with the STROBE reporting checklist (available at http://dx.doi. org/10.21037/jtd-20-3276).

\section{Methods}

\section{Study population and design}

Patients who underwent isolated LTx followed by posttransplant LINX implantation at Duke University Hospital between 2017 and 2019 were followed prospectively in the Reflux Following Lung Transplantation and Associated Treatment Registry. Follow-up was closed on September 3, 2020. Patients were referred by pulmonary transplant providers to thoracic surgery for evaluation of anti-reflux surgery and then reviewed and assessed by the surgical team. Based on review of physiologic test results, clinical history, and patient preference, patients were offered LINX placement and/or traditional fundoplication. Specifically, patients with normal esophageal motility and no dysphagia were given the option of either LINX placement or fundoplication. Those who preferred to undergo LINX placement subsequently underwent an insurance approval process to determine whether their insurance providers would cover the LINX device; patients whose insurance providers covered the device underwent LINX placement. Alternatively, patients with abnormal esophageal motility, those who preferred to undergo traditional fundoplication, and those who did not have insurance coverage for the LINX device underwent fundoplication. A subset of patients who underwent pre-transplant GERD evaluation were determined to have GERD severe enough to warrant early anti-reflux surgery following LTx based on results of ambulatory $\mathrm{pH}$ testing and barium swallow, and additional clinical findings including presence of bronchoscopic or histologic evidence of aspiration. These patients were triaged to anti-reflux surgery within 3 months of LTx without further post-transplant GERD evaluation. Patients who underwent prior fundoplication or alternative antireflux procedure were excluded from this registry. The study was conducted in accordance with the Declaration of Helsinki (as revised in 2013). The study was approved by the institutional review board of Duke University (Pro00058718) and informed consent was taken from all individual participants.

Patient demographics, medical history, and operative 
characteristics pertaining to LINX implantation were abstracted from patient charts. Medical history of interest included pre-transplant comorbidities (hypertension, diabetes, tobacco use, GERD), transplant type (single, bilateral), and donor and recipient characteristics including lung allocation score at transplantation, panel reactive antibody at transplantation, cytomegalovirus serostatus, and induction and maintenance immunosuppression. During the study period, LINX implantation was performed by a single surgeon specializing in foregut and lung transplant surgery.

Patients were followed by thoracic surgery and transplant providers at routine clinic visits per standard of care. All patients underwent baseline ambulatory 24-hour $\mathrm{pH}$ testing, high-resolution impedance esophageal manometry, and pulmonary function testing (PFT) prior to LINX implantation. During the study period, ambulatory $\mathrm{pH}$ testing was conducted using both dual probe $\mathrm{pH}$ tests and $\mathrm{pH}$ tests with impedance. Patients' $\mathrm{pH}$ test results including acid contact times and DeMeester scores were evaluated in the context of the listed reference ranges for the particular test administered to distinguish normal from abnormal results. Gastric emptying studies, upper endoscopy, and barium swallow were performed at the providers' discretion. After LINX implantation, follow-up data was recorded at the post-operative visit, 3, 6, and 12 months post-implantation. Use of acid-suppressing medication (PPI or H2 blocker), any episodes of redo LTx or GERDrelated reoperation, and recent PFTs were recorded at each follow-up time point and transbronchial lung biopsies were performed per clinical protocol. Unplanned clinic visits during the study period were recorded and adverse events were documented and reviewed. Patients were scheduled for repeat ambulatory $\mathrm{pH}$ testing and esophageal manometry as needed based on patient willingness, symptoms, and provider assessments. Date of last follow-up, patient status (alive or deceased), and dates and pathologic grades of any biopsy-proven acute rejection episodes were recorded.

\section{Comparison of LINX and traditional fundoplication}

A group of LTx recipients who underwent post-transplant LARS was matched 1:1 (nearest neighbor, caliper $=0.31$ ) based on age at anti-reflux surgery $( \pm 5$ years $)$ and sex. One-year outcomes and change in pulmonary function were compared between LTx recipients who underwent LINX implantation and those who underwent traditional fundoplication.

\section{Statistical analysis}

First, we characterized LTx recipients who underwent LINX implantation and evaluated the efficacy of the LINX device. Patient demographics, medical history, and operative characteristics were summarized using medians and interquartile ranges (IQR) for continuous variables and frequencies and proportions for categorical variables. Patient demographics, medical history, and anti-reflux surgery operative characteristics were compared between LINX and fundoplication groups using Wilcoxon rank-sum tests for continuous variables and Chi-squared and Fisher's exact tests for categorical variables. Among LINX patients, pre- and post-LINX data points were compared using statistical methods for paired data including McNemar's test for categorical variables (i.e., acid-suppressing medication use) and two-sample paired student's $t$ tests for continuous variables (i.e., distal acid contact time on ambulatory $\mathrm{pH}$ testing).

Second, we compared LTx recipients who underwent LINX implantation and those who underwent traditional LARS and evaluated the safety of the LINX device. Oneyear patient, rejection-free, side effect-free, and reoperationfree survival were estimated in an unadjusted fashion using the Kaplan-Meier method and compared between groups using log-rank tests. Characteristics of acute rejection episodes before and after anti-reflux surgery were compared between LINX and fundoplication groups using Chisquared and Fisher's exact tests. Pulmonary function was assessed based on the change in forced expiratory volume in one second $\left(\mathrm{FEV}_{1}\right)$ using a linear mixed effects model (fixed effects: time, procedure type; random effects: patient) with an interaction between time and procedure type to evaluate whether the change in FEV1 over time differed between LINX and fundoplication groups. A two-sided P value less than 0.05 was considered statistically significant. All analyses were performed using R version 3.6.1 (Vienna, Austria).

\section{Results}

\section{Study population}

Overall, 17 LTx recipients who underwent post-transplant LINX implantation were included. Median age at LINX implantation was 61 years (IQR, 44-71 years), 29.4\% were female, and $100 \%$ were White. Most $(76.5 \%)$ patients had undergone bilateral LTx, 11 (64.7\%) had a history of pre-transplant GERD, and 12 (70.6\%) were 
Table 1 Patient and lung transplant characteristics

\begin{tabular}{|c|c|c|c|}
\hline Characteristic $^{a}$ & LINX (N=17) & $\begin{array}{l}\text { FUNDO } \\
(\mathrm{N}=17)\end{array}$ & $\mathrm{P}$ value \\
\hline \multicolumn{4}{|l|}{ Demographics } \\
\hline \multicolumn{4}{|l|}{ Age (years) } \\
\hline \multirow[t]{2}{*}{ At anti-reflux surgery } & $\begin{array}{c}60.7 \\
{[44.4,70.5]}\end{array}$ & 60.8 & $>0.9$ \\
\hline & & {$[44.3,69.6]$} & \\
\hline At transplant & $\begin{array}{c}59.9 \\
{[41.6,64.9]}\end{array}$ & $\begin{array}{c}60.6 \\
{[44.2,69.1]}\end{array}$ & 0.7 \\
\hline Sex (female) & $5(29.4 \%)$ & $5(29.4 \%)$ & $>0.9$ \\
\hline Race (White) & $17(100 \%)$ & $16(94.1 \%)$ & 0.3 \\
\hline Ethnicity (Hispanic) & $0(0 \%)$ & $0(0 \%)$ & $>0.9$ \\
\hline \multicolumn{4}{|l|}{ Pre-transplant comorbidities } \\
\hline Hypertension & $6(35.3 \%)$ & $5(29.4 \%)$ & 0.7 \\
\hline Diabetes & $4(23.5 \%)$ & $3(17.6 \%)$ & 0.7 \\
\hline $\begin{array}{l}\text { Gastroesophageal reflux } \\
\text { disease }\end{array}$ & $11(64.7 \%)$ & $8(47.1 \%)$ & 0.3 \\
\hline Tobacco use & $7(41.2 \%)$ & $9(52.9 \%)$ & 0.5 \\
\hline Smoking pack-years ${ }^{b}$ & $\begin{array}{c}54.0 \\
{[23.0,60.0]}\end{array}$ & $\begin{array}{c}40.0 \\
{[31.3,46.9]}\end{array}$ & 0.6 \\
\hline $\begin{array}{l}\text { Acid-suppressing } \\
\text { medication use }\end{array}$ & $12(70.6 \%)$ & $10(58.8 \%)$ & 0.5 \\
\hline Proton pump inhibitor ${ }^{c}$ & $10(83.3 \%)$ & $10(100 \%)$ & \\
\hline $\mathrm{H} 2$ blocker $^{c}$ & $2(16.7 \%)$ & $0(0 \%)$ & \\
\hline $\begin{array}{l}\text { History of prior reflux } \\
\text { procedure }\end{array}$ & $0(0 \%)$ & $0(0 \%)$ & $>0.9$ \\
\hline \multicolumn{4}{|c|}{ Lung transplant characteristics } \\
\hline Transplant type & & & 0.7 \\
\hline Single & $4(23.5 \%)$ & $5(29.4 \%)$ & \\
\hline Bilateral & $13(76.5 \%)$ & $12(70.6 \%)$ & \\
\hline $\begin{array}{l}\text { Lung allocation score at } \\
\text { transplant }\end{array}$ & $\begin{array}{c}42.8 \\
{[40.6,45.4]}\end{array}$ & $\begin{array}{c}42.4 \\
{[36.8,55.9]}\end{array}$ & $>0.9$ \\
\hline Missing & $1(5.9 \%)$ & $0(0.0 \%)$ & \\
\hline \multicolumn{4}{|c|}{ Panel reactive antibody at transplant (\%) } \\
\hline Class I & $\begin{array}{c}0.00 \\
{[0.00,0.00]}\end{array}$ & $\begin{array}{c}0.00 \\
{[0.00,7.00]}\end{array}$ & 0.2 \\
\hline Class II & $\begin{array}{c}0.00 \\
{[0.00,2.00]}\end{array}$ & $\begin{array}{c}0.00 \\
{[0.00,0.00]}\end{array}$ & 0.2 \\
\hline \multicolumn{4}{|c|}{ Cytomegalovirus serostatus } \\
\hline Donor positive & $10(58.8 \%)$ & $11(64.7 \%)$ & 0.7 \\
\hline Recipient positive & $10(58.8 \%)$ & $6(35.3 \%)$ & 0.2 \\
\hline Mismatch (D+/R-) & $5(29.4 \%)$ & $6(35.3 \%)$ & 0.7 \\
\hline
\end{tabular}

Table 1 (continued)
Table 1 (continued)

\begin{tabular}{lccc}
\hline Characteristic $^{\text {a }}$ & LINX (N=17) & $\begin{array}{c}\text { FUNDO } \\
(\mathrm{N}=17)\end{array}$ & P value \\
\hline \multicolumn{2}{l}{ Induction immunosuppression } & 0.5 \\
None & $1(5.9 \%)$ & $0(0 \%)$ & \\
Corticosteroids & $2(11.8 \%)$ & $5(29.4 \%)$ & \\
Basiliximab & $14(82.4 \%)$ & $12(70.6 \%)$ & \\
Maintenance immunosuppression & & \\
Corticosteroids & $17(100 \%)$ & $17(100 \%)$ & $>0.9$ \\
Cyclosporine & $2(11.8 \%)$ & $0(0 \%)$ & 0.15 \\
Tacrolimus & $15(88.2 \%)$ & $17(100 \%)$ & 0.15 \\
Mycophenolate mofetil & $13(76.5 \%)$ & $15(88.2 \%)$ & 0.4 \\
Azathioprine & $3(17.6 \%)$ & $2(11.8 \%)$ & 0.6 \\
Other & $2(11.8 \%)$ & $0(0 \%)$ & 0.15 \\
\hline
\end{tabular}

a , continuous variables presented as median (interquartile range); , among patients with history of tobacco use; ' , among patients taking acid-suppressing medication. LINX, LINX Reflux Management System; FUNDO, fundoplication; D+, donor cytomegalovirus positive; $\mathrm{R}-$, recipient cytomegalovirus negative.

taking acid-suppressing medication prior to LTx. Patient and LTx characteristics were similar between LINX and fundoplication groups (Table 1).

\section{Operative characteristics of the LINX implantation}

LINX implantation was performed median 1.40 years (IQR, 0.52-2.73 years) post-transplant. Six (35.3\%) patients were found to have a hiatal hernia, most of which were documented as "small" (4 out of 6 patients). All procedures were performed laparoscopically; robotassistance was employed in $3(17.6 \%)$ cases. Two (11.8\%) patients underwent paraoesophageal hernia repair and 1 (5.9\%) underwent lysis of adhesions concurrent with LINX implantation. Median operative duration was 106 minutes (IQR, 102-139 minutes) and patients remained in the hospital for median 1 day (range, 1-4 days) post-operatively. No intraoperative complications were noted (Table 2).

Compared to LINX implantation, laparoscopic fundoplication cases were more likely to be performed with robot-assistance $(88.2 \%$ vs. $17.6 \%, \mathrm{P}<0.01)$. Postoperative hospital lengths of stay were significantly longer after fundoplication than after LINX implantation [median 2 (range 1-25) vs. 1 (range 1-4) days, $\mathrm{P}=0.02$ ]. Additional operative characteristics were similar between groups (Table 2). 
Table 2 Operative characteristics of LINX implantation versus traditional laparoscopic anti-reflux surgery

\begin{tabular}{|c|c|c|c|}
\hline Characteristic & LINX $(\mathrm{N}=17)$ & FUNDO $(\mathrm{N}=17)$ & $P$ value \\
\hline Hiatal hernia & $6(35.3 \%)$ & $3(17.6 \%)$ & 0.3 \\
\hline \multicolumn{4}{|l|}{ LINX size } \\
\hline 13 & 2 (11.8\%) & & \\
\hline 14 & $5(29.4 \%)$ & & \\
\hline 15 & 5 (29.4\%) & & \\
\hline 16 & 4 (23.5\%) & & \\
\hline 17 & $1(5.9 \%)$ & & \\
\hline
\end{tabular}

Surgical approach

\begin{tabular}{|c|c|c|c|}
\hline Laparoscopic & $17(100 \%)$ & $17(100 \%)$ & $>0.9$ \\
\hline Robot-assisted & $3(17.6 \%)$ & $15(88.2 \%)$ & $<0.01$ \\
\hline $\begin{array}{l}\text { Concomitant procedure } \\
\text { performed }\end{array}$ & $3(17.6 \%)$ & $2(11.8 \%)$ & 0.6 \\
\hline $\begin{array}{l}\text { Paraoesophageal hernia } \\
\text { repair }\end{array}$ & $2(11.8 \%)$ & $0(0 \%)$ & \\
\hline Lysis of adhesions & $1(5.9 \%)$ & $1(5.9 \%)$ & \\
\hline Pyloroplasty & $0(0 \%)$ & $1(5.9 \%)$ & \\
\hline Procedure length (minutes) & $\begin{array}{c}106 \\
{[102,139]}\end{array}$ & $\begin{array}{c}124 \\
{[104,180]}\end{array}$ & 0.3 \\
\hline $\begin{array}{l}\text { Post-operative length of } \\
\text { stay (days) }\end{array}$ & $\begin{array}{c}1.00 \\
{[1.00,1.00]}\end{array}$ & $\begin{array}{c}2.00 \\
{[1.00,3.00]}\end{array}$ & 0.02 \\
\hline Procedural complications & $0(0 \%)$ & $0(0 \%)$ & $>0.9$ \\
\hline
\end{tabular}

Continuous variables presented as median (interquartile range). Italic $P$ values indicate statistically significant findings with $\mathrm{P}<0.05$. LINX, LINX Reflux Management System; FUNDO, fundoplication.

\section{Efficacy of the LINX device}

\section{Ambulatory pH testing}

Pre-LINX ambulatory $\mathrm{pH}$ testing was performed median 0.21 years (range, -3.53 to 7.16 years) post-transplant; pretransplant $\mathrm{pH}$ tests were used for baseline assessment in three cases as these patients were considered to have severe GERD on pre-transplant evaluation that warranted an early post-LTx GERD surgery. Eight (47.1\%) patients underwent followup $\mathrm{pH}$ testing median 0.62 years (range, $0.19-1.04$ years) post-LINX implantation. Overall, 4 out of 8 (50.0\%) patients had a reduction in total distal acid contact time, 3 out of $8(37.5 \%)$ achieved normal total distal acid contact times, and 3 out of $8(37.5 \%)$ achieved normal DeMeester scores after LINX implantation [pre-LINX: 1/17 (5.9\%) and 2/17 (11.8\%), respectively] (Figures 1,2, Table 3). At one year post-LINX implantation, 14 (82.4\%) patients remained on acid-suppressing medication at the discretion of their pulmonary transplant teams (pre-LINX: $100 \%, \mathrm{P}=0.3$ ).

\section{Esophageal manometry}

Pre-LINX esophageal manometry was performed median 0.25 years (range, $0.10-7.17$ years) post-transplant. Nine $(52.9 \%)$ patients underwent follow-up esophageal manometry median 0.65 years (range, $0.19-1.04$ years) postLINX implantation. There were no significant changes in manometric parameters after LINX implantation (Table 4).

\section{Safety of the LINX device compared to traditional fundoplication}

Among patients who underwent LINX implantation, one-year patient, rejection-free, side effect-free, and reoperation-free survival were $94.1 \%, 70.6 \%, 29.4 \%$, and $94.1 \%$, respectively. Patient, rejection-free, and reoperation-free survival were similar between LINX and fundoplication groups (all $\mathrm{P}>0.05$ ). Side effect-free survival was worse among patients who underwent LINX implantation compared to those who underwent traditional fundoplication $(\mathrm{P}=0.02)$ (Figure 3). However, in general, side effects occurred early after LINX implantation and resolved over time. The most common side effects were minor including dysphagia, vomiting, residual reflux symptoms, and throat pain. One patient underwent device dilation for persistent dysphagia 55 days post-LINX implantation with subsequent device explant 77 days postimplantation for refractory dysphagia. Patients in both LINX and fundoplication groups experienced postoperative weight fluctuations. By 6 months postoperatively, patients who underwent LINX implantation experienced a median change in body weight of $+2.0 v s .-2.7 \mathrm{~kg}$ among those who underwent traditional fundoplication $(\mathrm{P}>0.05)$. Side effects that occurred among LINX and fundoplication patients during the first year after anti-reflux surgery are summarized in Table 5.

Fourteen (82.4\%) patients who underwent LINX implantation and $10(58.8 \%)$ patients who underwent traditional fundoplication had evidence of acute rejection on at least one biopsy prior to anti-reflux surgery $(\mathrm{P}=0.13)$. After anti-reflux surgery, 5 (29.4\%) LINX and 8 (47.1\%) fundoplication patients had at least one rejection episode within one year $(\mathrm{P}=0.3)$. The severity of rejection after anti-reflux surgery was graded as minimal (grade A1) or 

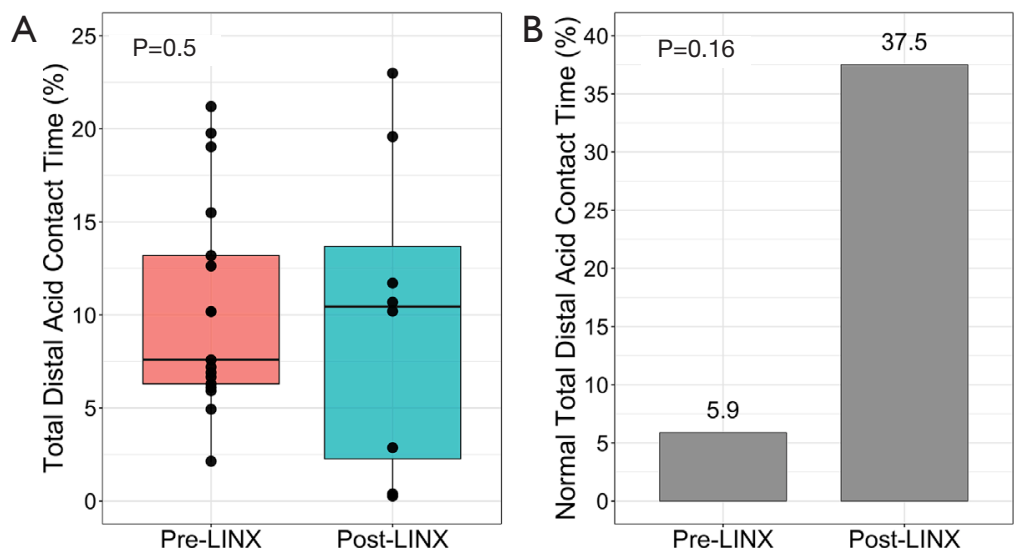

Figure 1 Total distal acid contact time before and after LINX implantation. (A) Distribution of total distal acid contact times. Each point represents a single patient who had ambulatory $\mathrm{pH}$ testing at each time point (pre-LINX: N=17; post-LINX: N=8). (B) Proportion of patients who demonstrated normal total distal acid contact time (pre-LINX: N=17; post-LINX: N=8). Normal total distal acid contact time was defined as $<4.2 \%$. LINX, LINX Reflux Management System.
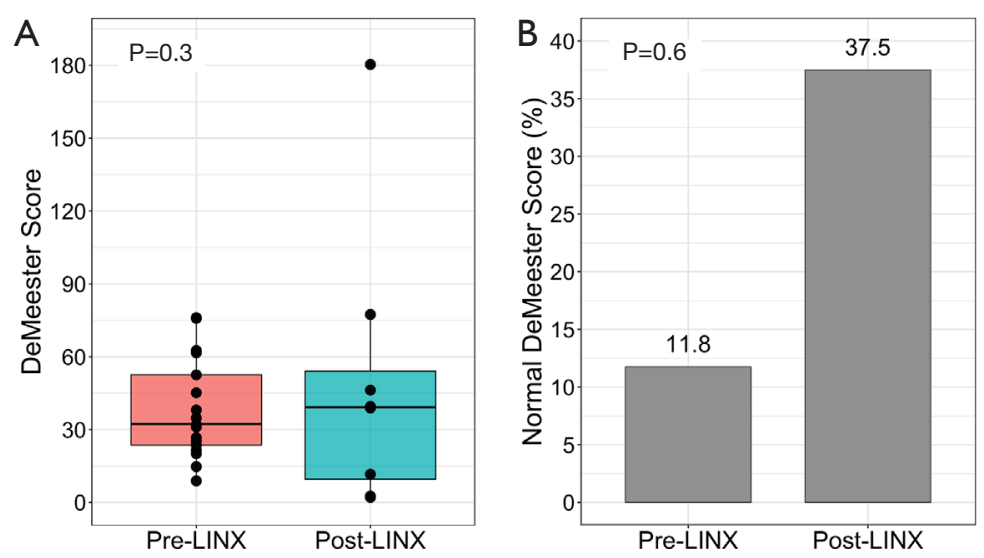

Figure 2 DeMeester score before and after LINX implantation. (A) Distribution of DeMeester scores. Each point represents a single patient who had ambulatory $\mathrm{pH}$ testing at each time point (pre-LINX: N=17; post-LINX: N=8). (B) Proportion of patients with a normal DeMeester score (pre-LINX: N=17; post-LINX: N=8). LINX, LINX Reflux Management System.

Table 3 Results of ambulatory $\mathrm{pH}$ testing before and after LINX implantation

\begin{tabular}{|c|c|c|c|c|c|c|}
\hline Characteristic & Pre-LINX (N) & Post-LINX (N) & Pre-LINX & Post-LINX & Median $\Delta$ & $P$ value \\
\hline Normal total distal acid contact time ${ }^{a}$ & 17 & 8 & $1(5.9 \%)$ & $3(37.5 \%)$ & & 0.16 \\
\hline DeMeester score & 17 & 8 & $32.3[23.6,52.6]$ & $39.2[9.55,54.1]$ & +0.55 & 0.3 \\
\hline Normal DeMeester score ${ }^{b}$ & 17 & 8 & $2(11.8 \%)$ & $3(37.5 \%)$ & & 0.6 \\
\hline
\end{tabular}

Continuous variables presented as median (interquartile range). ${ }^{a}$, normal total distal acid contact time $<4.2 \%$; ${ }^{b}$, normal DeMeester score is test dependent. Patient scores were evaluated based on the normal range for each individual test (dual probe $\mathrm{pH}$ test vs. $\mathrm{pH}$ test with impedance). Median $\Delta$, median change post-LINX implantation (compared to pre-LINX measurements). LINX, LINX Reflux Management System. 
Table 4 Results of esophageal manometry before and after LINX implantation

\begin{tabular}{|c|c|c|c|c|c|c|}
\hline Characteristic & Pre-LINX (N) & Post-LINX (N) & Pre-LINX & Post-LINX & Median $\Delta$ & $P$ value \\
\hline $\begin{array}{l}\text { Distal contractile integral } \\
\text { (mmHg-cm-s) }\end{array}$ & 17 & 9 & $\begin{array}{c}2,615.0 \\
{[1,082.5,3,999.2]}\end{array}$ & $\begin{array}{c}1,832.0 \\
{[1,600.0,2,752.0]}\end{array}$ & +614.0 & 0.4 \\
\hline
\end{tabular}

Continuous variables presented as median (interquartile range). Median $\Delta$, median change post-LINX implantation (compared to pre-LINX measurements). LINX, LINX Reflux Management System.
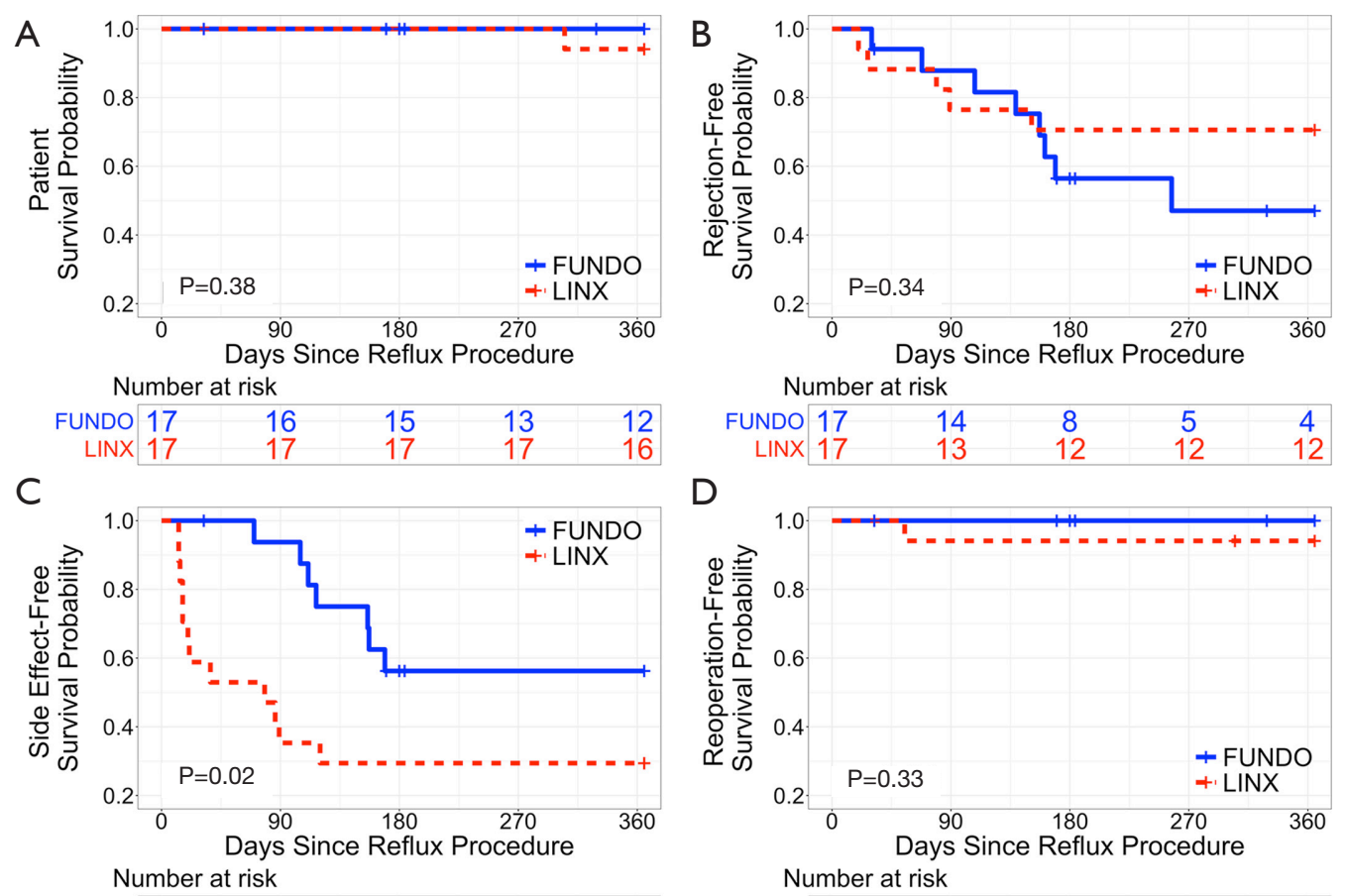

Figure 3 Comparison of one-year patient and adverse event-free survival between lung transplant recipients who underwent LINX implantation or traditional fundoplication. (A) Patient survival; (B) rejection-free survival; (C) side effect-free survival; (D) reoperation-free survival. LINX, LINX Reflux Management System.

mild (grade A2) in all cases (Table 6). There was no change in FEV1 across pre- and post-operative measurements in either LINX or fundoplication groups (Figure 4). The trajectory of FEV1 over time was similar between groups (interaction $\mathrm{P}=0.4$ ).

\section{Discussion}

In this study, we explored the safety and early efficacy of the LINX Reflux Management System, a novel implantable anti-reflux device, in LTx recipients. Compared to traditional LARS, the short-term safety of LINX implantation was similar with comparable rates of mortality, acute rejection, and reintervention up to one year postoperatively. As expected from post-market approval studies in non-transplant patients, LINX implantation was associated with significantly more early side effects, primarily dysphagia. In a limited efficacy assessment, a small subset of patients achieved normal esophageal acid exposure time after LINX implantation. Our findings suggest that use of the LINX Reflux Management System in this chronically immunosuppressed population is safe, but further study 
Table 5 Side effects during the first year after anti-reflux surgery

\begin{tabular}{lcc}
\hline Side effect & LINX $(\mathrm{N}=17)$ & FUNDO (N=17) \\
\hline Overall & & \\
At least one side effect & $12(70.6 \%)$ & $8(47.1 \%)$ \\
Individual events & & \\
Dysphagia & $11(64.7 \%)$ & $1(5.9 \%)$ \\
Nausea or vomiting & $5(29.4 \%)$ & $0(0 \%)$ \\
Reflux symptoms & $3(17.6 \%)$ & $3(17.6 \%)$ \\
Throat pain & $2(11.8 \%)$ & $0(0 \%)$ \\
Belching & $1(5.9 \%)$ & $0(0 \%)$ \\
Esophageal spasm & $2(11.8 \%)$ & $0(0 \%)$ \\
Inability to belch & $0(0 \%)$ & $1(5.9 \%)$ \\
Inability to vomit & $0(0 \%)$ & $3(17.6 \%)$ \\
Bloating & $0(0 \%)$ & $1(5.9 \%)$ \\
Abdominal pain or & $2(11.8 \%)$ & $1(5.9 \%)$ \\
cramping & & $0(0 \%)$ \\
Shortness of breath & & \\
Weight fluctuations & &
\end{tabular}

Before anti-reflux surgery $27.5[23.9,30.7] 26.1$ [23.6, 27.0]

6 months after anti-reflux $27.6[23.7,30.7] 26.6$ [24.1, 28.4] surgery

Weight change $(\mathrm{kg})^{\mathrm{b}}$ $+2.0[-1.1,+3.4]-2.7[-3.9,+2.3]$

a continuous variables presented as median (interquartile range); ${ }^{b}$, before versus 6 months after anti-reflux surgery. LINX, LINX Reflux Management System; FUNDO, fundoplication.

Table 6 Comparison of biopsy-proven acute rejection episodes before and after anti-reflux surgery

\begin{tabular}{lccc}
\hline Characteristic & $\begin{array}{c}\text { LINX } \\
(\mathrm{N}=17)(\%)\end{array}$ & $\begin{array}{c}\text { FUNDO } \\
(\mathrm{N}=17)(\%)\end{array}$ & P value \\
\hline $\begin{array}{l}\text { At least one acute rejection } \\
\text { episode before anti-reflux } \\
\text { surgery }\end{array}$ & $14(82.4)$ & $10(58.8)$ & 0.13 \\
$\begin{array}{l}\text { At least one acute rejection } \\
\text { episode within one year after } \\
\text { anti-reflux surgery }\end{array}$ & $5(29.4)$ & $8(47.1)$ & 0.3 \\
$\begin{array}{l}\text { Pathologic grade of post- } \\
\text { operative acute rejection }\end{array}$ & & & \\
A1 (minimal) & & & \\
A2 (mild) & $4(80.0)$ & $7(87.5)$ & \\
\hline LINX, LINX Reflux Management System; FUNDO, fundoplication.
\end{tabular}
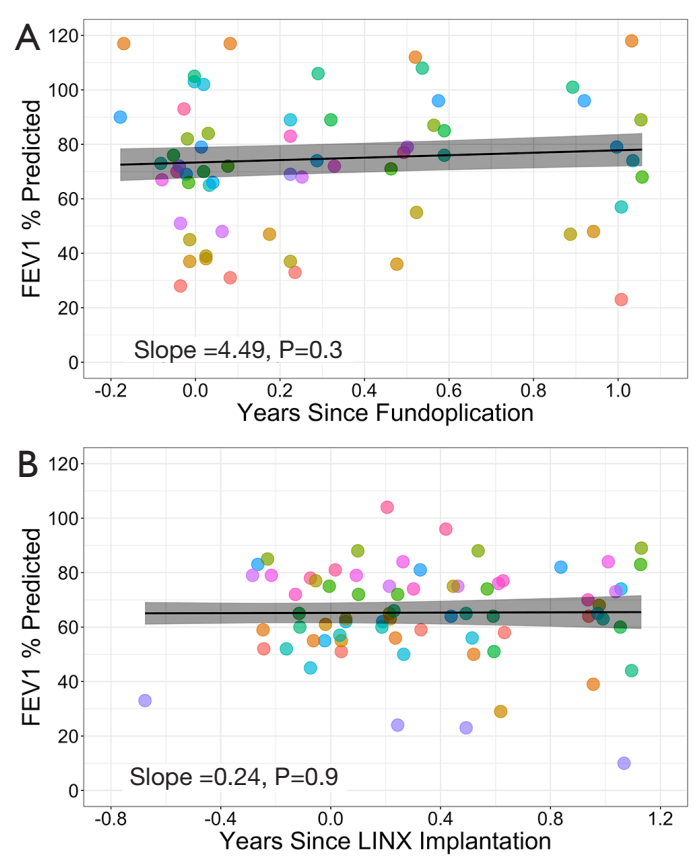

Figure 4 Change in forced expiratory volume in one second (FEV1) over one year after anti-reflux surgery. Time zero represents the date of anti-reflux surgery. Therefore, negative years represent measurements taken before and positive years represent measurements taken after anti-reflux surgery. Points correspond to actual patient measurements and lines correspond to the best fit determined from a linear mixed effects model. (A) Change in forced expiratory volume in one second among lung transplant recipients who underwent traditional fundoplication. (B) Change in forced expiratory volume in one second among lung transplant recipients who underwent LINX implantation. LINX, LINX Reflux Management System.

is required to understand its long-term effectiveness for surgical management of post-transplant GERD.

The efficacy of the LINX device among patients in the general population has previously been reported with outcomes up to 5 years post-implantation detailed in the literature $(19,22)$. As the foundational study in this area, Bonavina et al. conducted a multicenter prospective clinical trial examining use of the LINX device for management of medication-refractory GERD and found that the majority of patients demonstrated a significant decrease in esophageal acid exposure by 3 months after LINX implantation (23). They have since reported outcomes at 1, 2, 4, and 5 years post-LINX implantation, demonstrating a sustained reduction in esophageal acid exposure time throughout the follow-up period with up to $90 \%$ of patients 
achieving normal esophageal acid exposure time by 2 years, maintained by $75 \%$ by 5 years $(16,18,19)$. These findings are corroborated by Ganz et al. who report normalization of esophageal acid exposure time in $58 \%$ and greater than or equal to $50 \%$ reduction of esophageal acid exposure time in $64 \%$ of patients by 1 year, the latter increasing to $83 \%$ of patients by 5 years $(22,24)$.

Since postoperative patients who are asymptomatic are less likely to submit to repeat ambulatory $\mathrm{pH}$ testing, post-LINX acid exposure times were available for only $37.5 \%$ of patients in our study. Nevertheless, within that group $50 \%$ had a reduction in total distal acid contact times, which is comparable to the studies referenced above. Also, while baseline total distal acid contact times were similar among patients in our study compared to those in the general population (median $7.6 \%$ vs. $8.0-11.9 \%$ ) $(16,18,19,23,24)$, the pathophysiology of GERD in LTx recipients is complex, affected by multiple factors including use of immunosuppressive medications and anatomic considerations such as vagal nerve injury resulting from the transplant operation that are absent among non-transplant patients $(1-3,25)$. In light of these unique circumstances that persist beyond LINX implantation, it may be unreasonable to expect that LTx recipients will achieve normal esophageal acid exposure times, particularly in the short term post-LINX period. Furthermore, while the majority of patients in our study continued acid-suppressing medication after LINX implantation despite resolution of GERD symptoms, this reflects standard practice among the transplant pulmonology providers at our institution, and therefore cannot be used as a reliable indicator of LINX efficacy in this population. This is further complicated by a significant proportion of LTx recipients with GERD who exhibit minimal or no symptoms. Continued long-term follow-up with acquisition of additional post-LINX $\mathrm{pH}$ testing is required to better characterize the effects of LINX implantation on esophageal acid exposure in these complex patients. Accordingly, our team has considered increasing use of BRAVO $\mathrm{pH}$ studies both pre- and postoperatively, since use of a small capsule rather than a $\mathrm{pH}$ probe may reduce patient discomfort and improve willingness to undergo testing at multiple timepoints.

Prior studies have also addressed the safety of the LINX device, particularly surrounding the concern for erosion of the device into the esophagus $(23,24)$. Despite this concern, device erosions and migrations seem to be rare with no occurrences reported during post-implantation follow-up across numerous studies $(16,18,19,22-24)$. At our institution, the LINX device is routinely upsized after measurement of the esophageal circumference. This leaves the device somewhat "loose" at the gastroesophageal junction, but we believe that subsequent scarring secures it in place while at the same time ensuring that device erosion does not occur.

A more legitimate concern post-LINX implantation is the potential for dysphagia. Across previous studies, dysphagia affects between $43-68 \%$ of patients early after implantation $(16,18,23,24)$. This problem tends to resolve over time decreasing to approximately $10 \%$ at 1 year and less than $5 \%$ beyond 2 years (24). Accordingly, persistent dysphagia was the most common indication for reintervention, with patients undergoing device dilation followed by device explant if dilation did not provide adequate symptom relief. Rates of device explant across prior studies ranged from $2-7 \%(16,18,19,22,23)$. These findings are reflected in our results, which indicate that dysphagia and vomiting were the most common side effects among patients in our cohort. Nevertheless, for most patients, postoperative dysphagia was transient, resolving spontaneously within one year after LINX implantation. In our study, only one individual (5.9\% of patients) underwent device dilation followed by device explant due to persistent dysphagia. That patient had normal manometry prior to implantation of the device; however, a barium swallow suggested mild to moderate esophageal dysmotility with associated tertiary contractions.

Consistent with prior studies, LINX patients at our institution are initiated on a regular diet immediately on postoperative day $1(16,18,19,23,24)$. In contrast, fundoplication patients begin with a liquid diet, transitioning to solids later in the recovery period. Indeed, prior work suggests that the LINX device may better support normal eating behavior during the early postoperative period (23), likely facilitating early weight gain among LINX patients in our cohort, compared to weight loss among those undergoing fundoplication. Likewise, in addition to adjusting to the device itself, the high rate of dysphagia associated with LINX implantation may be confounded by differential postoperative management protocols for patients undergoing distinct anti-reflux procedures. Importantly, however, prior work suggests that a central benefit of MSA devices as an alternative to traditional LARS is preserved ability to belch and vomit (16). Indeed, fewer than $5 \%$ of patients reported inability to belch or vomit after LINX implantation (18), compared to 34\% of dissatisfied post-fundoplication patients (26). Although we did not specifically examine this aspect of post-LINX 
quality of life and esophageal function in our study, inability to belch or vomit were not specifically noted among LINX patients in our cohort, but were among the most common side effects reported in the fundoplication group, affecting $17.6 \%$ of patients during the first post-operative year.

While long-term use of the LINX device has been associated with a satisfactory safety profile within the general population, one primary concern surrounding longterm device implantation in LTx patients is risk of severe infection in the face of chronic immunosuppression. In an institutional study of LTx recipients, Palmer et al. found that post-transplant survival was significantly compromised among patients with documented bacteremia during the post-transplant period, with 44\% 3-year survival among patients with an episode of bacteremia versus $71 \%$ among those without $(\mathrm{P}=0.0001)$ (21). In the present study, there were no documented cases of infection or bacteremia. Once implanted, the LINX device is not exposed to the environment, which may reduce the long-term risk of infection associated with this device. While our findings support the short-term safety of LINX implantation in LTx recipients, a longer duration of follow-up is needed to fully understand the safety of LINX implantation in this population.

There are several limitations in our study that warrant discussion. First, this study examines a small cohort of patients at a single, large academic institution and therefore represents a highly specialized experience that may not be generalizable to other institutions. As additional technologies to treat GERD become more widely utilized, follow-up studies should examine trends in a larger group of LTx recipients that may likewise be better equipped to compare efficacy among novel anti-reflux technologies and traditional LARS procedures. Additionally, within our cohort only $47.1 \%$ and $52.9 \%$ of patients underwent follow-up ambulatory $\mathrm{pH}$ testing and esophageal manometry, respectively. While follow-up tests were scheduled for the remaining patients, all cancelled their appointments for reasons including hospitalization, other illness, and patient request. As testing was not rescheduled in any of these cases, we may reasonably infer that patients for whom follow-up testing was not conducted were doing well, without cause to return for evaluation with what are well-recognized as particularly uncomfortable tests. Our assessment of the efficacy of the LINX device among LTx recipients may therefore be biased in favor of patients who underwent follow-up testing deemed necessary by patient or provider assessment and may not have captured those who achieved entirely favorable results. Further study with rigorous acquisition of follow-up $\mathrm{pH}$ testing and esophageal manometry is required to test this hypothesis and further elucidate the efficacy of the LINX device in LTx recipients; in the future, increased utilization of BRAVO $\mathrm{pH}$ studies may reduce patient discomfort and increase the proportion of patients who are amenable to follow-up testing. Likewise, a subset of patients in our study who underwent early posttransplant anti-reflux surgery due to the presence of severe GERD on pre-transplant evaluation, did not undergo repeat post-transplant $\mathrm{pH}$ testing prior to LINX implantation. As esophageal acid exposure may change considerably after LTx, future studies should ensure that all patients, regardless of pre-transplant GERD severity, undergo repeat post-transplant $\mathrm{pH}$ testing prior to anti-reflux surgery to facilitate better understanding of the expected change in esophageal acid exposure time associated with post-LTx LINX implantation. Finally, the short duration of postLINX implantation follow-up in this study limits our ability to comment on the true safety of the LINX device. While our findings offer important new insight into the potential utility of the LINX device in LTx recipients, demonstrating safety comparable to that observed in the first year after traditional fundoplication in addition to highlighting the potential to hasten postoperative recovery through shorter postoperative hospital stays and prompt return to normal dietary habits, additional studies are needed to assess long-term outcomes of this relatively new technology in chronically immunosuppressed populations. Specifically, while our results suggest that pulmonary function was unchanged across pre- and post-LINX measurements, the short duration of follow-up in this study limits our ability to provide robust examination of the pre- and postLINX incidence and prevalence of CLAD, the prevention of which remains a leading reason for aggressive surgical management of GERD in LTx recipients. As a sustained decline in $\mathrm{FEV}_{1}$ to $\leq 80 \%$ of baseline for at least 3 weeks is required to diagnose "probable" CLAD, or at least 3 months to diagnose "confirmed" CLAD (4), ongoing follow-up beyond one year is required to better elucidate the trajectory of FEV1 post-LINX implantation and understand the ability of the LINX device to prevent or improve CLAD among treated patients. Likewise, as new techniques for surgical management of post-transplant GERD continue to evolve, future studies should explore the safety and efficacy of the LINX device in comparison to other alternative anti-reflux procedures including the Stretta procedure and transoral incisionless fundoplication. 


\section{Conclusions}

To our knowledge, this is the first study to examine the use of novel implantable devices for management of post-transplant GERD in LTx recipients. Use of the LINX Reflux Management System in a cohort of LTx recipients at our institution was associated with an overall favorable short-term safety profile compared to traditional fundoplication, and preserved pulmonary function up to one year post-operatively. However, only a small subset of patients achieved reduced or normal esophageal acid exposure times. While our findings suggest that the LINX Reflux Management System is a safe method for surgical management of post-LTx GERD, further investigation is needed to understand its efficacy and long-term safety in this chronically immunosuppressed population.

\section{Acknowledgments}

Funding: This work was supported by the National Institutes of Health [TL1TR002555 to SEH and AYC], 5T32HL069749 to OKJ], and Torax Medical, Inc. The content is solely the responsibility of the authors and does not necessarily represent the official views of the National Institutes of Health, or Torax Medical, Inc.

\section{Footnote}

Reporting Checklist: The authors have completed the STROBE reporting checklist. Available at http://dx.doi. org/10.21037/jtd-20-3276

Data Sharing Statement: Available at http://dx.doi. org/10.21037/jtd-20-3276

Conflicts of Interest: All authors have completed the ICMJE unifor disclosure form (available at http://dx.doi. org/10.21037/jtd-20-3276). MGH reports grants from Torax, during the conduct of the study. The other authors have no conflicts of interest to declare.

Ethical Statement: The authors are accountable for all aspects of the work in ensuring that questions related to the accuracy or integrity of any part of the work are appropriately investigated and resolved. The study was conducted in accordance with the Declaration of Helsinki (as revised in 2013). The study was approved by the institutional review board of Duke University
(Pro00058718) and informed consent was taken from all individual participants.

Open Access Statement: This is an Open Access article distributed in accordance with the Creative Commons Attribution-NonCommercial-NoDerivs 4.0 International License (CC BY-NC-ND 4.0), which permits the noncommercial replication and distribution of the article with the strict proviso that no changes or edits are made and the original work is properly cited (including links to both the formal publication through the relevant DOI and the license). See: https://creativecommons.org/licenses/by-nc-nd/4.0/.

\section{References}

1. Young LR, Hadjiliadis D, Davis RD, et al. Lung Transplantation Exacerbates Gastroesophageal Reflux Disease. Chest 2003;124:1689-93.

2. Davis CS, Shankaran V, Kovacs EJ, et al. Gastroesophageal reflux disease after lung transplantation: Pathophysiology and implications for treatment. Surgery 2010;148:737-44; discussion 744-5.

3. Hadjiliadis D, Davis RD, Steele MP, et al. Gastroesophageal reflux disease in lung transplant recipients. Clin Transplant 2003;17:363-8.

4. Verleden GM, Glanville AR, Lease ED, et al. Chronic lung allograft dysfunction: Definition, diagnostic criteria, and approaches to treatment-A consensus report from the Pulmonary Council of the ISHLT. J Heart Lung Transplant 2019;38:493-503.

5. Hartwig MG, Appel JZ, Li B, et al. Chronic aspiration of gastric fluid accelerates pulmonary allograft dysfunction in a rat model of lung transplantation. J Thorac Cardiovasc Surg 2006;131:209-17.

6. Li B, Hartwig MG, Appel JZ, et al. Chronic aspiration of gastric fluid induces the development of obliterative bronchiolitis in rat lung transplants. Am J Transplant 2008;8:1614-21.

7. D'Ovidio F, Mura M, Ridsdale R, et al. The effect of reflux and bile acid aspiration on the lung allograft and its surfactant and innate immunity molecules SP-A and SP-D. Am J Transplant 2006;6:1930-8.

8. Fisichella PM, Davis CS, Lundberg PW, et al. The protective role of laparoscopic antireflux surgery against aspiration of pepsin after lung transplantation. Surgery 2011;150:598-606.

9. Hartwig MG, Duane Davis R. Gastroesophageal reflux disease-induced aspiration injury following 
lung transplantation. Curr Opin Organ Transplant 2012;17:474-8.

10. Fisichella PM, Davis CS, Kovacs EJ. A review of the role of GERD-induced aspiration after lung transplantation. Surg Endosc 2012;26:1201-4.

11. Hartwig MG, Anderson DJ, Onaitis MW, et al. Fundoplication After Lung Transplantation Prevents the Allograft Dysfunction Associated With Reflux. Ann Thorac Surg 2011;92:462-8; discussion 468-9.

12. Lau CL, Palmer SM, Howell DN, et al. Laparoscopic antireflux surgery in the lung transplant population. Surg Endosc 2002;16:1674-8.

13. Palmer SM, Miralles AP, Howell DN, et al. Gastroesophageal reflux as a reversible cause of allograft dysfunction after lung transplantation. Chest 2000;118:1214-7.

14. Davis RD, Lau CL, Eubanks S, et al. Improved lung allograft function after fundoplication in patients with gastroesophageal reflux disease undergoing lung transplantation. J Thorac Cardiovasc Surg 2003;125:533-42.

15. Robertson AGN, Krishnan A, Ward C, et al. Anti-reflux surgery in lung transplant recipients: Outcomes and effects on quality of life. Eur Respir J 2012;39:691-7.

16. Bonavina L, Demeester T, Fockens P, et al. Laparoscopic sphincter augmentation device eliminates reflux symptoms and normalizes esophageal acid exposure: One-and 2-year results of a feasibility trial. Ann Surg 2010;252:857-62.

17. Du X, Hu Z, Yan C, et al. A meta-analysis of long followup outcomes of laparoscopic Nissen (total) versus Toupet $\left(270^{\circ}\right)$ fundoplication for gastro-esophageal reflux disease based on randomized controlled trials in adults. BMC Gastroenterol 2016;16:88.

18. Lipham JC, DeMeester TR, Ganz RA, et al. The LINX ® reflux management system: Confirmed safety and efficacy now at 4 years. Surg Endosc 2012;26:2944-9.

Cite this article as: Halpern SE, Gupta A, Jawitz OK, Choi AY, Salfity HV, Klapper JA, Hartwig MG. Safety and efficacy of an implantable device for management of gastroesophageal reflux in lung transplant recipients. J Thorac Dis 2021;13(4):2116-2127. doi: $10.21037 /$ jtd-20-3276
19. Bonavina L, Saino G, Bona D, et al. One hundred consecutive patients treated with magnetic sphincter augmentation for gastroesophageal reflux disease: 6 years of clinical experience from a single center. J Am Coll Surg 2013;217:577-85.

20. Asti E, Bonitta G, Lovece A, et al. Longitudinal comparison of quality of life in patients undergoing laparoscopic Toupet fundoplication versus magnetic sphincter augmentation: Observational cohort study with propensity score analysis. Medicine (Baltimore) 2016;95:e4366.

21. Palmer SM, Alexander BD, Sanders LL, et al. Significance of blood stream infection after lung transplantation: analysis in 176 consecutive patients. Transplantation 2000;69:2360-6.

22. Ganz RA, Edmundowicz SA, Taiganides PA, et al. Long-term Outcomes of Patients Receiving a Magnetic Sphincter Augmentation Device for Gastroesophageal Reflux. Clin Gastroenterol Hepatol 2016;14:671-7.

23. Bonavina L, Saino GI, Bona D, et al. Magnetic augmentation of the lower esophageal sphincter: Results of a feasibility clinical trial. J Gastrointest Surg 2008;12:2133-40.

24. Ganz RA, Peters JH, Horgan S, et al. Esophageal sphincter device for gastroesophageal reflux disease. N Engl J Med 2013;368:719-27.

25. Hoppo T, Jarido V, Pennathur A, et al. Antireflux surgery preserves lung function in patients with gastroesophageal reflux disease and end-stage lung disease before and after lung transplantation. Arch Surg 2011;146:1041-7.

26. Humphries LA, Hernandez JM, Clark W, et al. Causes of dissatisfaction after laparoscopic fundoplication: The impact of new symptoms, recurrent symptoms, and the patient experience. Surg Endosc 2013;27:1537-45. 